\title{
Signatures of restarted activity in core-dominated, triple radio sources selected from the FIRST survey
}

\author{
A. Marecki ${ }^{1}$, P. Thomasson ${ }^{2}$, K.-H. Mack ${ }^{3}$, and M. Kunert-Bajraszewska ${ }^{1}$ \\ 1 Toruń Centre for Astronomy, N. Copernicus University, 87-100 Toruń, Poland \\ e-mail: amr@astro.uni.torun.pl \\ 2 Jodrell Bank Observatory, The University of Manchester, Macclesfield, Cheshire, SK11 9DL, UK \\ 3 INAF - Istituto di Radioastronomia, via Gobetti 101, 40129 Bologna, Italy
}

Received 5 August 2005 / Accepted 21 October 2005

\section{ABSTRACT}

\begin{abstract}
Signatures of the re-occurrence of activity in radio-loud AGNs, indicated either by the so-called double-double or X-shaped structures, have been observed in a number of radio sources. All such objects known to date have linear sizes of the order of a megaparsec. A number of the sources that are appreciably more compact than this, but that exhibit hints of a past phase of activity, were found in the VLA FIRST survey. Their structures show symmetric relic lobes straddling relatively bright, unresolved cores. Observations of the cores of 15 such structures with MERLIN at $5 \mathrm{GHz}$ have shown that four of them are doubles or core-jets on the subarcsecond scale. Misalignments of $\Delta \mathrm{PA} \geqslant 30^{\circ}$ between the axis of the inner structure and the line connecting the fitted maxima of the arcminute-scale relic lobes are clearly visible in three of the four sources. From these results, we can infer that a rapid repositioning of the central engine in each of these three radio sources is the most plausible interpretation of the observed morphology and that a merger is most likely the original cause of such a repositioning. In the case of TXS 1033+026, the optical image extracted from the SDSS archives clearly suggests that two objects separated by only $2.7 \mathrm{kpc}$ (projected onto the sky plane) are indeed merging. The inner parts of TXS $0818+214$ and TXS $1312+563$ could be interpreted as double-lobed, and consequently, these sources could be of the double-double type; but further multifrequency observations are necessary to provide support for such an interpretation.
\end{abstract}

Key words. radio continuum: galaxies - galaxies: active

\section{Introduction}

Firm observational evidence now exists that a galaxy, regardless of its state of activity, hosts a supermassive black hole (SMBH, see e.g. Barth 2003, and references therein). If a significant amount of matter in the vicinity of the SMBH accretes onto it, the galaxy appears to be active, but for how long does this activity occur? Can an inactive galaxy become active and vice versa, and is repeated switching between active and inactive states possible? Radio observations can perhaps give some answers to these questions because the lobes in radioloud AGNs partly provide a historical record of past activity. From detailed measurements of the spectral indices of their lobes, the spectral ages of extended radio sources can be inferred that, according to Alexander \& Leahy (1987) and Liu et al. (1992), are of the order of $\sim 10^{7}$ to $10^{8}$ years. However, as discussed in detail by Scheuer (1995), double-lobed radio sources are known to expand with velocities about a few percent of the speed of light; consequently the overall linear sizes of these sources must be of the order of $10^{5}$ to $10^{6} \mathrm{pc}$. This agrees with observations and the fact that some radio galaxies - they are termed Giant Radio Galaxies (GRGs) - may have sizes $\geq 1 \mathrm{Mpc}^{1}$. As the spectral ages of radio galaxies, even GRGs, are thought to be two orders of magnitude less than their actual ages, which, in turn, can be about the same age as the Universe (Pasquini et al. 2004), it would appear that activity phenomena are episodic: they can be started at an arbitrary stage in the evolution of the galaxy and can cease or become interrupted after a certain period of time.

If the energy supply from the central engine to the hotspots and the lobes of a radio-loud AGN cuts off, it enters a socalled "coasting phase". It can remain at this stage of its evolution for up to $10^{8}$ years, and its spectrum would become very steep due to radiation and expansion losses during this period (Komissarov \& Gubanov 1994; Slee et al. 2001). On the other hand, as first pointed out by Hargrave \& Ryle (1974), the radiative lifetimes of the relativistic electrons in the hotspots are of

\footnotetext{
${ }^{1}$ For consistency with numerous papers on GRGs (e.g. Schoenmakers et al. 1999, 2000a,b,c; Kaiser et al. 2000; Lara et al. 2001) $H_{0}=50 \mathrm{~km} \mathrm{~s}^{-1} \mathrm{Mpc}^{-1}$ and $q_{0}=0.5$ is used throughout this paper. For these cosmological parameters the redshift value of $z=1.25$ provides the maximum linear size for a given angular size: $1^{\prime \prime}$ corresponds to $8.6 \mathrm{kpc}$.
} 
the order of only $10^{4}$ years. Therefore, the lack of fuelling in radio sources that have switched off - they are sometimes termed "faders" - should result in the absence of the hotspots as they should have faded away relatively quickly. A few objects of this kind have been observed in samples of ultra-steep spectrum sources (Röttgering et al. 1994; De Breuck et al. 2000) or as a part of very low frequency surveys (Cohen et al. 2004; Mack et al. 2005).

Although it is obvious that total disappearance should normally be the ultimate stage in the evolution of a fader, it is also clear that under certain circumstances a new period of activity could begin during the "coasting phase", i.e. before the lobes have faded out completely. An observable effect of this would be the presence of new, bright components located in or straddling the centre of a larger, double-lobed relic structure. The signature of such renewed activity is most convincing if it takes the form of a smaller, also double-lobed radio source giving rise to a so-called double-double radio galaxy - DDRG (Lara et al. 1999; Schoenmakers et al. 2000a,b). According to the theory developed by Kaiser et al. (2000) - hereafter KSR - one of the most noticeable features of DDRGs is that they are large-size radio objects ( $\gtrsim 700 \mathrm{kpc}$ ) because recurrent activity cannot be observed in radio structures that are significantly smaller than GRGs. The reason for this is that $\sim 5 \times 10^{7}$ years are required to refill the cocoon with matter from warm clouds of gas embedded in the hot Intergalactic Medium (IGM) passing through the bow shock surrounding the outer source structure. Otherwise, the gas densities within the cocoon are insufficient for the development of new shocks/hotspots.

In spite of this, an attempt was made to search for signatures of restarted activity in radio sources with sizes less than those of GRGs. This is important because, if DDRGs with linear sizes considerably below $1 \mathrm{Mpc} d o$ exist, modification of the theory of KSR would appear inevitable. In Sect. 2 a procedure is presented to search for candidates that are potentially restarted objects with sizes below $1 \mathrm{Mpc}$. Using this procedure a small sample of such objects was constructed and observed with MERLIN at $5 \mathrm{GHz}$ (Sect. 3). The results are discussed in Sect. 4 and summarised in Sect. 5.

\section{A search for restarted objects in the FIRST survey}

Radio sources with extended emission, whose structures have been clearly imaged in the Faint Images of Radio Sky at Twenty centimetres (FIRST) survey (White et al. 1997) ${ }^{2}$, have angular sizes of the order of a few arcminutes and therefore linear sizes less than $\approx 1 \mathrm{Mpc}$, regardless of their redshifts. Consequently, an initial systematic search through the FIRST catalogue for triple sources with a dominant core would yield objects with sizes less than $1 \mathrm{Mpc}$, which could form a list of candidates for objects showing signatures of restarted activity.

An automatic procedure was developed for this. In the first phase of this procedure, a source within FIRST with a flux density greater than a particular limit $(75 \mathrm{mJy})$ was selected and a pair of "secondary" sources (the "lobes") were sought

\footnotetext{
${ }^{2}$ Official website: http://sundog.stsci.edu
}

within a $2^{\prime}$ radius of the initially selected source (the "core"). An aligned triple was assumed to have been detected if the angle lobe ${ }_{1}-$ core - lobe $_{2}$ was greater than $165^{\circ}$ and the peak flux densities of the "lobes" were less than $30 \%$ of that of the "core". This was repeated for all the sources in FIRST with flux densities that were greater than the limit. The $30 \%$ threshold was somewhat arbitrary, but was based upon an initial, extensive visual inspection of FIRST maps: the peak flux densities of the lobes in the vast majority of sources with the required morphology fell well below that limit anyway. No lower limit for the flux density of the FIRST components of relic lobes was imposed. The sources of this kind are labelled core-dominated triples (CDTs) throughout this paper.

As a next step, 5-GHz flux densities of the selected sources were obtained from the GB6 survey (Becker et al. 1991), and those sources with flux densities below $40 \mathrm{mJy}$ were rejected. Also rejected were those whose spectral indices calculated from the FIRST and GB6 flux densities were: $\alpha<0.5$ $\left(S \propto v^{-\alpha}\right)$. The 5-GHz flux density limit was adopted to ensure that high signal to noise images of the "cores" would result from MERLIN follow-up observations at that frequency. (The aforementioned $75 \mathrm{mJy}$ limit at $1.4 \mathrm{GHz}$ resulted from the 5-GHz flux density limit and the spectral index criterion.) The sample was limited to objects with steep spectra to maximise the probability that the "cores" themselves, which mainly contribute to the total flux density, were likely to have steep spec$\operatorname{tra}^{3}$. Generic core-jet sources, which typically have flat spectra, were possibly eliminated in this way.

This automated procedure was carried out using the version of the FIRST catalogue dated 15 October 2001 (the most recent at the time the project began) covering approximately 7954 square degrees in the north Galactic cap and 611 square degrees in the south Galactic cap, and containing $\sim 771000$ sources. It follows that with the GB6 covering only the northern hemisphere, our sample is also limited in the same manner. It yielded 313 sources; their maps were extracted from the FIRST archive and inspected visually. It turned out that the majority of the alleged triple structures were typically compact objects surrounded by symmetric artefacts originating from the VLA diffraction pattern that is typical of snapshot mode observations. These were rejected and a final sample of 15 sources resulted from the whole selection process. Their names, coordinates, flux densities at $1.4 \mathrm{GHz}$ and $4.85 \mathrm{GHz}$, and spectral indices between these two are shown in Table 1.

The approach described above is somewhat complementary to the one of Schoenmakers et al. (2000c) and Lara et al. (2001). These authors searched for GRGs in WENSS and NVSS, respectively, and their selection criteria included a lower limit to the sources' angular sizes, which was $5^{\prime}$ and $4^{\prime}$, respectively. As mentioned in Sect. 1, in this study, objects that are less extended than GRGs are being investigated and so an upper limit of $4^{\prime}$ has been adopted.

After MERLIN observations of the 15 targets (reported in Sect. 3) had been made, four sources of greatest interest were chosen for more detailed analysis. In Table 2 the flux

\footnotetext{
3 Obviously, calculation of the spectral indices of the "cores" alone was not possible because of the resolution limit of the GB6 survey.
} 
Table 1. Sources observed using MERLIN at $5 \mathrm{GHz}$.

\begin{tabular}{|c|c|c|c|c|c|c|}
\hline \multirow{3}{*}{$\begin{array}{c}\text { Source } \\
(1)\end{array}$} & RA & \multirow[t]{2}{*}{ Dec } & \multicolumn{2}{|c|}{ Integrated flux at } & \multirow{3}{*}{$\begin{array}{l}\text { Spectral } \\
\text { index } \\
(6)\end{array}$} & \multirow{3}{*}{$\begin{array}{l}\text { Sub-arcsecond } \\
\text { structure } \\
\text { (7) }\end{array}$} \\
\hline & $(\mathrm{J} 2000)$ & & $1.4 \mathrm{GHz}$ & $4.85 \mathrm{GHz}$ & & \\
\hline & $(2)$ & (3) & (4) & $(5)$ & & \\
\hline TXS 0726+256 & 072905.744 & +253034.24 & 136.40 & 63 & 0.64 & single \\
\hline TXS 0818+214 & 082107.501 & +211703.06 & 187.55 & 56 & 1.00 & Figure 1 \\
\hline TXS 0940+001 & 094319.237 & -000425.08 & 1158.61 & 499 & 0.70 & single \\
\hline TXS 1002+554 & 100617.423 & +551244.98 & 169.80 & 62 & 0.83 & single \\
\hline TXS 1024+549 & 102734.175 & +544240.25 & 118.29 & 48 & 0.75 & undetected \\
\hline TXS 1025+089 & 102833.021 & +083957.12 & 103.18 & 40 & 0.78 & undetected \\
\hline TXS $1033+026$ & 103631.933 & +022145.53 & 235.71 & 120 & 0.56 & Figure 2 \\
\hline TXS 1046+187 & 104853.954 & +182937.65 & 90.68 & 46 & 0.56 & marginally detected \\
\hline TXS 1308+011 & 131121.170 & +005319.23 & 257.97 & 98 & 0.80 & Figure 4 \\
\hline TXS 1309+484 & 131211.144 & +480925.22 & 165.55 & 85 & 0.55 & single \\
\hline TXS $1312+563$ & 131458.416 & +560342.00 & 364.00 & 155 & 0.71 & Figure 5 \\
\hline FIRST J152523.6+053736 & 152523.639 & +053735.94 & 233.34 & 113 & 0.60 & single \\
\hline FIRST J155726.1+360133 & 155726.130 & +360133.24 & 136.27 & 45 & 0.92 & marginally detected \\
\hline FIRST J162544.9+271929 & 162544.872 & +271229.39 & 293.30 & 110 & 0.81 & single \\
\hline B3 $1704+437$ & 170624.098 & +434030.37 & 123.33 & 45 & 0.84 & single \\
\hline
\end{tabular}

Description of the columns: Col. (1): source name; Col. (2): right ascension (J2000) extracted from FIRST; Col. (3): declination (J2000) extracted from FIRST; Col. (4): the sum of the fluxes densities of FIRST components; Col. (5): GB6 flux density; Col. (6): spectral index calculated using data from Cols. 4 and 5; Col. (7): sub-arcsecond structure as imaged using MERLIN at $5 \mathrm{GHz}$ and reported in this paper - see the figures and notes in Sect. 3.2.

Table 2. Positions and $1.4 \mathrm{GHz}$ flux densities of the components of four sources with nontrivial sub-arcsecond structures as indicated by the FIRST catalogue.

\begin{tabular}{cccccc}
\hline \hline Source & RA & Dec & \multicolumn{2}{c}{$S$ [mJy] } & Distance from \\
& \multicolumn{2}{c}{$(\mathrm{J} 2000)$} & Peak & \multicolumn{1}{c}{ Total } & the "core" ["] \\
\hline \multirow{3}{*}{ TXS 0818+214 } & 082106.547 & +211719.26 & 6.92 & 23.25 & 21.0 \\
& 082107.501 & +211703.06 & 142.20 & 148.32 & - \\
& 082108.357 & $+21 \quad 1649.09$ & 6.12 & 15.98 & 18.4 \\
& & & & & \\
TXS 1033+026 & 103628.166 & +022228.88 & 4.15 & 4.11 & 71.2 \\
& 103631.933 & +022145.53 & 186.11 & 202.43 & - \\
& 103633.024 & +022136.80 & 21.32 & 27.92 & 18.5 \\
& & & & & \\
TXS 1308+011 & 131120.744 & +005310.70 & 28.22 & 51.67 & 10.7 \\
& 131121.170 & +005319.23 & 134.20 & 144.57 & - \\
& 1311.786 & +005329.46 & 39.40 & 61.73 & 13.8 \\
& 131457.908 & +560332.46 & 18.54 & 28.08 & 10.4 \\
& 131457.987 & +560346.06 & 2.39 & 20.69 & 5.4 \\
& 131458.416 & +560342.00 & 270.24 & 276.44 & - \\
& 131459.232 & +560352.20 & 23.95 & 34.39 & 12.3 \\
\hline
\end{tabular}

densities and locations of the FIRST components of these four are quoted from the FIRST catalogue.

\section{Observations}

\subsection{Overview}

The MERLIN full-track, phase-referenced observations at $5 \mathrm{GHz}$ were carried out for the sample of 15 sources during the period 16 Feb.-1 Jun. 2004. 3C 286 was used as the flux density and polarisation calibrator, and OQ208 was used as the point source calibrator. The flux density of $3 \mathrm{C} 286$ on the VLA scale, which was used, was 7.331 Jy. The initial editing and calibration of the data were carried out using the Jodrell Bank d-programmes, and preliminary phase-referenced images were produced using the AIPS based PIPELINE procedure developed at Jodrell Bank. Further cycles of phase self-calibration and imaging using the AIPS tasks CALIB and IMAGR were then used to produce the final images.

As indicated in Table 1, two of the five weakest sources of the sample with GB6 flux densities below $50 \mathrm{mJy}-$ TXS 1024+549 and TXS 1025+089 - were not detected at the $3 \sigma$ level, corresponding to $430 \mu \mathrm{Jy} /$ beam and $320 \mu \mathrm{Jy} /$ beam, 

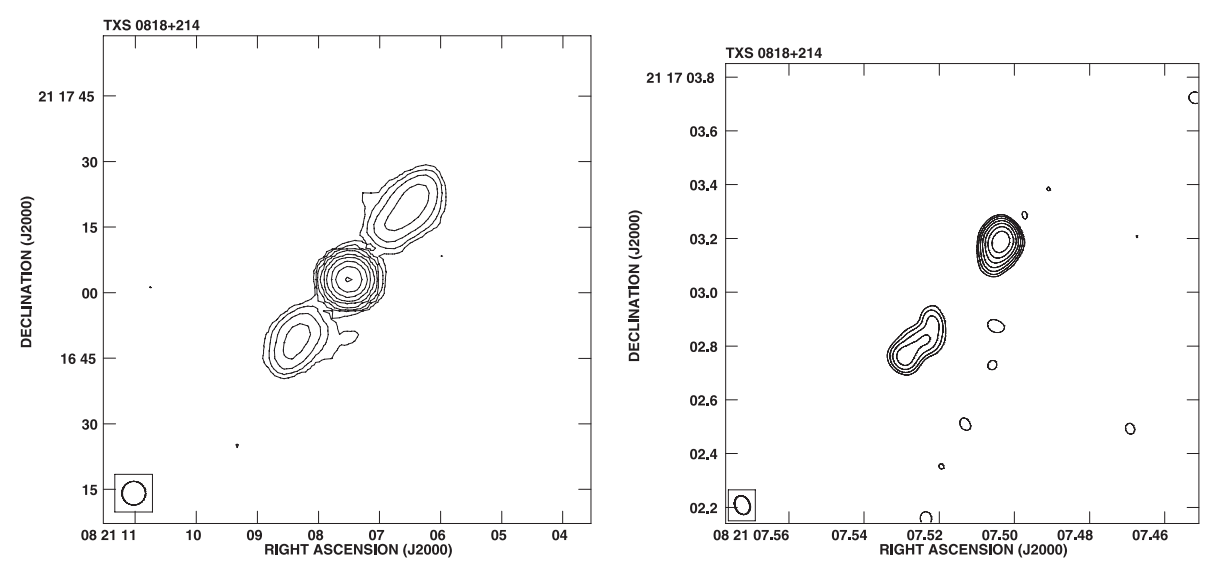

Fig. 1. TXS 0818+214. Left panel: FIRST map. Contours increase by a factor of 2; the first contour level is at $0.5 \mathrm{mJy} / \mathrm{beam}$. Right panel: MERLIN map at $5 \mathrm{GHz}$. Contours increase by a factor of 2 ; the first contour level corresponds to $\approx 5 \sigma$ level, which is $0.23 \mathrm{mJy} / \mathrm{beam}$. The peak flux density is $27.3 \mathrm{mJy} /$ beam. The beam size is $72 \times 54$ milliarcsec at a position angle of $21^{\circ}$.

Table 3. Fitted parameters of the MERLIN map components.

\begin{tabular}{cccr}
\hline \hline $\begin{array}{c}\text { Source } \\
\text { name } \\
(1)\end{array}$ & RA & $\begin{array}{c}\text { Dec } \\
(2)\end{array}$ & $\begin{array}{r}S_{5 \mathrm{GHz}} \\
{[\mathrm{mJy}]} \\
(4)\end{array}$ \\
\hline TXS 0818+214 & 082107.504 & 211703.18 & 39.9 \\
& 082107.526 & 211702.80 & 9.0 \\
TXS 1033+026 & 103631.946 & 022145.54 & 11.4 \\
& 103631.938 & 022145.41 & 7.5 \\
TXS 1308+011 & 131121.157 & 005319.47 & 15.2 \\
& 131121.161 & 005319.26 & 39.3 \\
TXS 1312+563 & 131458.405 & 560342.43 & 78.8 \\
& 131458.412 & 560341.81 & 46.6 \\
\hline
\end{tabular}

Description of the columns: Col. (1): source name; Col. (2): component right ascension (J2000) estimated using JMFIT; Col. (3): component declination (J2000) estimated using JMFIT; Col. (4): flux density (mJy) obtained using JMFIT.

respectively. Another two of these five - TXS 1046+187 and FIRST J155726.1+360133 - were only marginally detected; i.e. some diffuse structures appear at the $3 \sigma$ level corresponding to $300 \mu \mathrm{Jy} / \mathrm{beam}$ and $390 \mu \mathrm{Jy} /$ beam, respectively. The lack of any distinct features in their MERLIN images is probably the result of the diffuse structures being resolved. Seven sources appeared to be unresolved or barely resolved. They are listed as "single" in Table 1 and will not be discussed further here. The remaining four targets, listed in Table 2, have well resolved structures consisting of more than one component. Their MERLIN images are presented and comments on them given in Sect. 3.2. The flux densities of the components shown in these images were measured using the AIPS task JMFIT and are listed in Table 3.

\subsection{Notes on individual sources}

TXS 0818+214. This object was included in Release 4 of the Sloan Digital Sky Survey (SDSS/DR4) 4 as a $m_{R}=21.73$ galaxy; but its redshift was not known, and it could not be found

\footnotetext{
${ }^{4}$ This is the most recent release at the time of writing.
}

in the literature. Based both on the $r-z$ Hubble diagram as derived by e.g. Thompson et al. (1994) and on the given magnitude, an estimated redshift would be $z \approx 1.0$. The MERLIN image of the "core" of the source (Fig. 1, right panel) shows it as a double located at PA $=-40^{\circ}$. Given that the two components are asymmetric - the northwestern one is appreciably more compact than the southeastern one - it could be that the subarcsecond-scale structure is a core-jet type. The approximate alignment between the subarcsecond-scale and arcminute-scale structures (Fig. 1) agrees with this. However, despite the compactness of the northwestern component, it and the southeastern part of the source could still be regarded as two FR II-type (Fanaroff \& Riley 1974) lobes. No measurable polarisation was observed with MERLIN. Thus, only a detailed study of their spectral indices based on the present data and future observations of the source at a lower frequency and with a similar resolution might determine which is the correct interpretation. However, if the two components are actually lobes and given that the redshift estimate for this source translates to a linear size $\$ 586 \mathrm{kpc}$, such a linear size would be somewhat less than the minimum for a double-double source required by the KSR model $(\approx 700 \mathrm{kpc})$. The sizes quoted above are not likely to be underestimated, as $586 \mathrm{kpc}$ would result from an assumption of $z=1.25$ and so it is the maximum possible linear size for the observed angular size $\left(68^{\prime \prime}\right)$ and the adopted cosmological model. Therefore, if the double-double morphology is confirmed, it would appear that the KSR model would need to be modified to become applicable to TXS 0818+214.

TXS 1033+026. Figure 3 shows a $1^{\prime} \times 1^{\prime}$ (approx.) cutout from the SDSS/DR4 archive centred on the brightest ("core") component of TXS 1033+026 seen in the FIRST image (Fig. 2, left panel). In the optical domain it is also the brightest feature in this field: a "fuzzy" object with two emission peaks in its very centre and an arc-like structure emerging from there towards the northeast and bent so that it eventually points to the southeast. There are also two regularly shaped galaxies in the field: SDSS J103631.50+022201.9 with $m_{R}=17.84$ located $18^{\prime \prime}$ to the north of the centre and SDSS J103633.24+022120.6 with $m_{R}=17.63$ located $32^{\prime \prime}$ to the southeast of the centre. Neither 

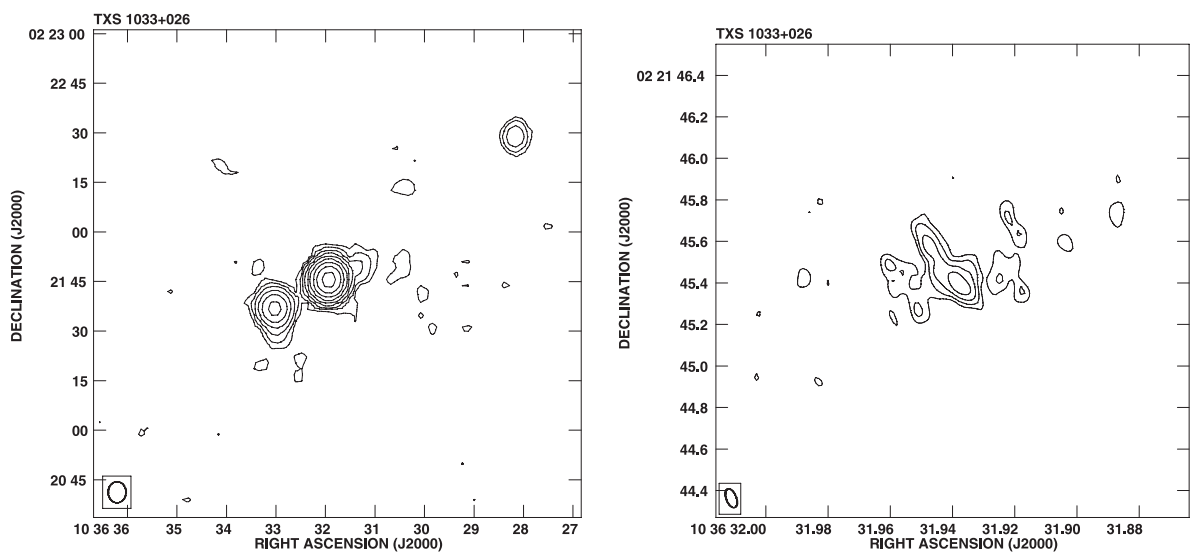

Fig. 2. TXS 1033+026. Left panel: FIRST map. Contours increase by a factor of 2 ; the first contour level is at $0.5 \mathrm{mJy} / \mathrm{beam}$. Right panel: MERLIN map at $5 \mathrm{GHz}$. Contours increase by a factor of 2 ; the first contour level corresponds to $\approx 3 \sigma$ level, which is $0.38 \mathrm{mJy} / \mathrm{beam}$. The peak flux density is $5.4 \mathrm{mJy} / \mathrm{beam}$. The beam size is $96 \times 48$ milliarcsec at a position angle of $21^{\circ}$.

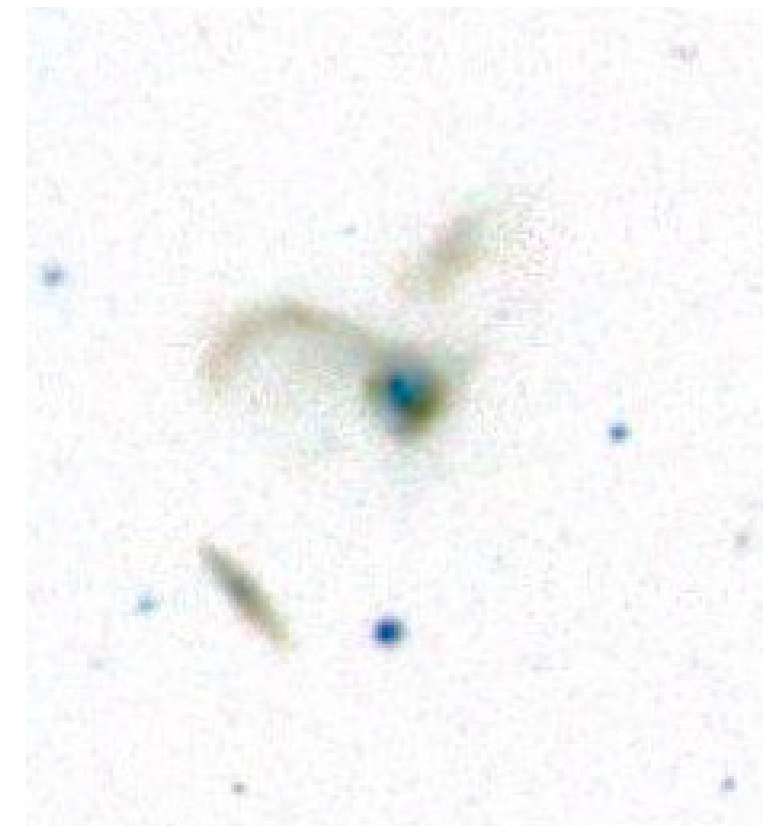

Fig. 3. Negative of the SDSS/DR4 optical image centred on the position of the radio source TXS $1033+026$. The size of the image is approximately $1^{\prime} \times 1^{\prime}$.

of these has a known redshift nor a radio counterpart in the FIRST image.

According to SDSS/DR4, one of the optical peaks, SDSS J103631.95+022145.7 (ObjId = 587726032776265858), is coincident within 0.' 4 with the "core" of the FIRST source, but its redshift is not listed there, nor could it be found in the literature. The second optical peak, SDSS J103631.87+022144.0 (ObjId $=587726032776265859)$, which is $1^{\prime \prime} .67$ off the position of the radio source, is a galaxy CGCG 037-089 whose redshift, $z=0.050276$, has been listed in the SDSS archive. At this redshift an angular distance of $2^{\prime \prime}$, which is the distance between the two optical maxima, corresponds to $2.7 \mathrm{kpc}$. At such a short (projected) distance, one would expect these two objects to be strongly interacting. Together with the presence of the arclike structure closely resembling e.g. those in the "Antennae" galaxies, the observed structure is perhaps evidence that a merger is taking place.

The arcminute-scale structure of the radio source as shown in the FIRST image (Fig. 2, left panel) could be viewed in more than one way. It could be considered as a very asymmetric triple with a central core, a lobe to the southeast, and a jet almost pointing in a northwesterly direction to a second lobe $\simeq 3.5$ times farther away from the core than the southeastern lobe. At the redshift given above, the projected size of the source would be $141 \mathrm{kpc}$. It could also be that the component located at RA $=10^{\mathrm{h}} 36^{\mathrm{m}} 28^{\mathrm{s}}$, Dec $=02^{\circ} 22^{\prime} 30^{\prime \prime}(\mathrm{J} 2000)$ is not associated with the main source, but is a coincidence. In this case, $1033+026$ could still be regarded as a CDT with the protrusion from the core to the northwest being the lobe. The linear size of the triple would then shrink to $72.6 \mathrm{kpc}$.

The principal features of the subarcsecond-scale structure seen in the MERLIN image (Fig. 2, right panel) are a core and a jet pointing to the northeast. In addition, the "remains" of flux to the southeast and to northwest of the core (i.e. the directions of the two lobes or lobe and jet visible in the FIRST image), which have been mostly resolved by MERLIN, are also visible. The jet is at an angle of $\approx 80^{\circ}$ relative to the direction of the southeastern lobe in the FIRST image. No measurable polarisation has been observed for this source with MERLIN.

TXS 1308+011. This source is identified with a QSO at a redshift $z=1.075$ (Hewett et al. 1995). The projected linear size is therefore $420 \mathrm{kpc}$. The MERLIN image (Fig. 4, right panel) indicates that in the subarcsecond scale the central component seen in the FIRST image is a core-jet type source with the northern component, which appears to be unresolved, being the core. The inner core-jet structure has a linear size of $3.8 \mathrm{kpc}$ with the jet initially pointing to the southeast at $\mathrm{PA} \approx-17^{\circ}$ and then bending away from its $55^{\circ}$ misalignment relative to the FIRST image structure towards a southwesterly direction, i.e. into the direction of the lobe in the FIRST image. Significant polarisation occurs where the jet is bending. There is no indication of a counter-jet to the north of the core, which is perhaps not visible as it is moving away from the observer. From the symmetry of the FIRST image, one might expect that any counter-jet would perhaps bend towards the northeastern 

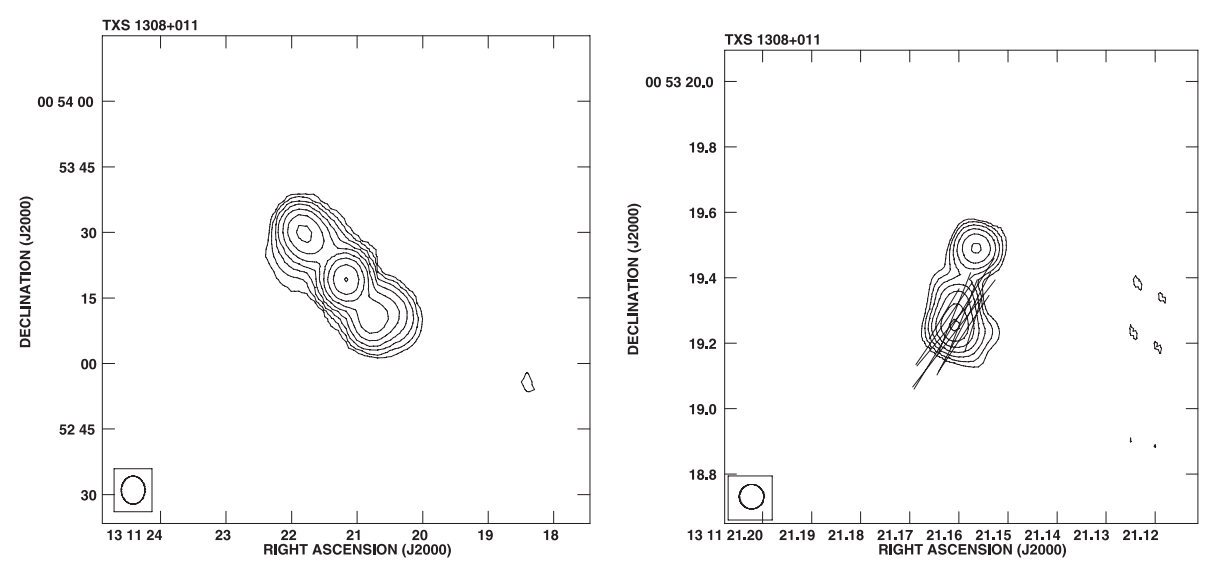

Fig. 4. TXS 1308+011. Left panel: FIRST map. Contours increase by a factor of 2; the first contour level is at $0.5 \mathrm{mJy} / \mathrm{beam}$. Right panel: MERLIN map at $5 \mathrm{GHz}$. Contours increase by a factor of 2 ; the first contour level corresponds to $\approx 3 \sigma$ level, which is $0.35 \mathrm{mJy} / \mathrm{beam}$. The peak flux density is $24.9 \mathrm{mJy} / \mathrm{beam}$. The polarisation line of 0 '. 5 amounts to $2.5 \mathrm{mJy} / \mathrm{beam}$. The circular beam size is $75 \mathrm{milliarcsec}$.
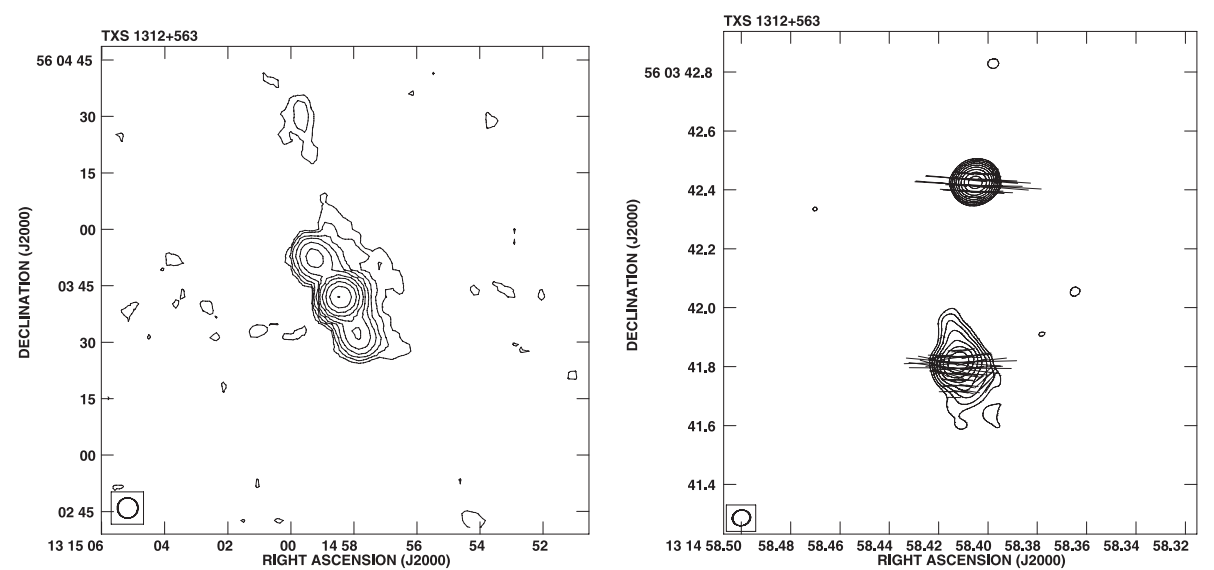

Fig. 5. TXS 1312+563. Left panel: FIRST map. Contours increase by a factor of 2; the first contour level is at $0.5 \mathrm{mJy} / \mathrm{beam}$. Right panel: MERLIN map at $5 \mathrm{GHz}$. Contours increase by a factor of 2 ; the first contour level corresponds to $\approx 5 \sigma$ level, which is $0.23 \mathrm{mJy} / \mathrm{beam}$. The peak flux density is $77.7 \mathrm{mJy} /$ beam. Polarisation line of 0.5 amounts to $2 \mathrm{mJy} /$ beam. The beam size is $60 \times 53$ milliarcseconds at a position angle of $-76^{\circ}$.

component. If all the morphological features suggested above were actually observed, TXS $1308+011$ could be regarded as an "Z-shaped" radio source analogous to TXS 1346+268 (also known as $4 \mathrm{C}+26.42$, van Breugel et al. 1984) and a helical jet or precessing jet model might be applied to it.

The phenomenon of a misalignment between the milliarcsecond- and arcsecond-scale structures has been thoroughly investigated; see Appl et al. (1996) for a review. Based on a large collection of observational material, they have concluded that, although it is possible to apply a helical jet model to individual sources, it is difficult to find a single mechanism responsible for the observed distribution of misalignments, which shows a statistically significant peak at $\triangle \mathrm{PA} \simeq 90^{\circ}$. This peak is particularly well-defined for BL Lac objects, but EVN and MERLIN observations of bent radio jets in some BL Lac objects (Cassaro et al. 2002) do not support a helical jet explanation of misalignments close to $90^{\circ}$. Thus, although an interpretation of the morphology based on a twisted jet model can be argued - it has been for example for TXS 1055+018 (Attridge et al. 1999) - adopting a scenario of a central engine repositioning leads to a simple and natural explanation without complex modelling. Therefore, the restarted activity scenario is not ruled out for TXS $1308+011$.

TXS 1312+563. According to SDSS/DR4, this source is identified with a QSO at a redshift $z=1.751$. The projected linear size is therefore $327 \mathrm{kpc}$. The MERLIN image (Fig. 5, right panel) of the arcminute-scale "core" component shows it as a double source located at $\mathrm{PA} \approx-5^{\circ}$ with a linear size of $8 \mathrm{kpc}$. The northern component is very compact and is not resolved by MERLIN, but the degree of its polarisation is appreciable: $2.8 \%$. The southern component resembles an FR II lobe and is strongly polarised $(7.1 \%)$ in the same direction as the northern one. As in the case of TXS $0818+214$, without spectral index data it is not possible to classify the inner structure of this source, which could be either of a core-lobe type as hinted by the morphology or a double-lobe type as suggested by the polarisation properties.

There is a $30^{\circ}$ misalignment of the subarcsecond-scale double relative to the arcminute-scale triple structure (Fig. 5, left panel). It should be noted that a very similar misalignment has been observed in the double-double GRG 1245+676 (Marecki et al. 2003). Thus, a hypothesis that TXS $1312+563$ could be 
of a double-double type cannot be ruled out, and if confirmed, the estimate for the linear size of TXS $1312+563$, which is well below the $\approx 700 \mathrm{kpc}$ limit required by the KSR, would indicate that their theory, unless modified, might not be applicable to this object.

\section{Discussion}

As already mentioned in Sect. 1, Lara et al. (1999) and Schoenmakers et al. (2000a,b) have identified a number of radio sources where the interruption of activity has had a spectacular impact on their double-double morphologies. J0116-47 (Saripalli et al. 2002), PKS B1545-321 (Saripalli et al. 2003), and SGRS J2159-7219 (Saripalli et al. 2005) are also clear examples of restarted DDRGs. Three other sources investigated by Saripalli et al. (2005) - SGRS J0143-5431, J0746-5702, and J1946-8222 - show evidence of either one- or two-sided knots/jets closer to the nucleus and might be examples of giant radio sources with relic lobes and restarting beams.

Another well-known type of a dramatic event in the evolution of a radio-loud AGN that may be labelled "restarted activity" can also lead to the development of an X-shaped radio source (Rottmann 2001, and references therein). It is to be noted that typical X-shaped sources, such as 3C 223.1 or 3C 403 (Dennett-Thorpe et al. 2002; Capetti et al. 2002), in principle resemble DDRGs like J0116-473 or PKS B1545-321 except for the misalignment between the active (inner) and the inactive (outer) parts. Thus, it might be speculated that the mechanism that triggered the development of the new structures in these two kinds of objects is the same, but that DDRGs are just "special cases" where the misalignment is (close to) zero. However, the opposite suggestion, namely that different mechanisms could be responsible for activity renewal in each type of object, appears to be more justified at the present time. A review of several possible mechanisms has been given by Schoenmakers et al. (2000a).

Mergers seem to be the most "natural" explanation of activity re-ignition, but if the angular momentum of the infalling gas is different from that of the pre-existing accretion disk, which is what would normally be expected ${ }^{5}$, a fast realignment of the jet axis might occur and an X-shaped source could emerge (KSR). Merritt \& Ekers (2002) show that the orientation of a black hole spin axis would change dramatically even in a minor merger, leading to an almost instantaneous reorientation of the coalescing SMBHs and a sudden flip in the direction of the jets. Liu (2004) suggests that the realignment of a rotating SMBH with a misaligned accretion disk is due to the Bardeen-Petterson effect (Bardeen \& Petterson 1975) and that the timescale of such a realignment is $<10^{5}$ years. It follows that the change of the direction of the jets is abrupt compared with the typical age of the radio sources (Komissarov \& Gubanov 1994).

\footnotetext{
5 Consequently, well-aligned DDRGs should be exceptional. However, Liu et al. (2003) point out that if the mass ratio of the two merging black holes is in the range 0.01 to 0.4 , realignment of the central engine and the subsequent formation of an X-shaped radio structure are not inevitable, and so the above-mentioned speculation that DDRGs can be regarded as "zero-misalignment" X-shaped sources could still be a viable scenario.
}

However, it is to be noted, that, although mergers seem to be the simplest explanation of activity re-ignition, an alternative model based upon the existence of thermal-viscous instabilities in the accretion disks of AGNs has been proposed (Hatziminaoglou et al. 2001, and references therein). According to Kunert-Bajraszewska et al. (2005), Marecki et al. (2006), and Kunert-Bajraszewska et al. (2006), this model could explain the existence of sources in which activity has ceased, but this does not preclude the possibility that the same mechanism could be responsible not only for a cessation but also for a re-ignition of activity.

The most outstanding feature of both DDRGs and $\mathrm{X}$-shaped sources is that they are large - their linear sizes exceed $700 \mathrm{kpc}$ - which indicates that they have to be particularly old for activity renewal regardless of their morphological features. The apparent lack of observable double-double structures in small-scale sources could mean that specific conditions are required for activity re-ignition, which do exist in GRGs, but which make it rare if not impossible for such a phenomenon to occur in more compact objects. It could be that the physical conditions inside the cocoon might not favour the development of the inner lobes for a long time after the initial burst of the activity. According to KSR, that timescale could be up to $\sim 5 \times 10^{7}$ years so that, even if the activity in a source younger than that could actually cease and than restart, such an event would remain unobservable.

While the above currently appears to be the case for DDRGs without exception, $\mathrm{X}$-shaped sources with linear sizes significantly below $1 \mathrm{Mpc}$ may exist. Two examples of such sources - TXS 0229+131 (Murphy et al. 1993) and 4C +01.30 (Wang et al. 2003) - can be found in the literature. The arms of TXS $0229+131(z=2.059)$ have lengths in the range $100-120 \mathrm{kpc}$. (The strange appearance of TXS 0229+131 could in principle result from gravitational lensing but according to Browne, priv. comm., it is very unlikely.) The "active" and "relic" arms of $4 \mathrm{C}+01.30(z=0.132)$ are 160 and $300 \mathrm{kpc}$ in length, respectively.

The conspicuous X-shaped structures observed in some $\mathrm{Mpc}$-scale radio sources and the two more compact ones mentioned above require a lack of almost any distortions caused by orientation effects. In other words, both arms of the cross must lie close to the sky plane for sources to be observed as such. If such an exceptional orientation is not present, Doppler boosting and beaming effects would distort the X-shaped structure considerably. In particular, if the arms pertinent to the "old" structures lie in the sky plane whereas the arms resulting from the renewed activity do not, the observed structure would be a superposition of an "old", double-lobed fader-like component and an asymmetric i.e. core-jet central component, which might not be perceived as such in low-resolution observations that are sufficient for a proper imaging of the double-lobed component. It follows that CDTs could actually be X-shaped sources distorted by beaming and Doppler boosting, and their morphologies are a consequence of a fast repositioning of the central engine. Recently, as a result of investigating $\mathrm{ZwCl} 0735.7+7421$, Cohen et al. (2005) have suggested that a realignment of a rotating SMBH followed by a repositioning of the accretion disk 
and the jets is a plausible interpretation of misaligned radio structures, even if they are not conspicuously X-shaped.

We conclude that it is plausible that three misaligned radio structures shown here in detail could be distorted X-shaped or $\mathrm{Z}$-shaped sources probably resulting from merger events. The existence of a merger in TXS $1033+026$ actually seems to be visible in the SDSS image. It is to be noted that the theory given by KSR, which precludes the possibility of the existence of aligned mini-DDRGs, can be reconciled with observations of misaligned restarted sources with outer structure sizes below $\approx 700 \mathrm{kpc}$. This is because the misalignments are usually large and the shapes of the cocoons highly non-spherical. In such circumstances, even if the parameters of the medium inside the cocoon prevent the development of inner lobes so that the secondary jets move "silently" across the cocoon's interior, they would shortly reach the side of the cocoon where they would be exposed to the unperturbed and much higher density IGM. Observable jets or hotspots could then appear.

\section{Conclusions and future work}

A programme to find radio sources with linear sizes below $1 \mathrm{Mpc}$ and showing signatures of possibly restarted activity was undertaken. To this end a computer-aided selection procedure aimed at finding CDTs in the FIRST catalogue was devised and a sample of candidates drawn up. These were then observed with MERLIN at $5 \mathrm{GHz}$ to reveal the subarcecond-scale morphology of their central ("core") components. In 4 out of 15 targets observed, the "cores" appear either as doubles or core-jets, with three of them misaligned with respect to their outer structures. Repositioning of the central engine of an AGN caused by a merger event seems to be a plausible scenario leading to the development of the observed morphologies in these three sources, as difficulties can arise with application of the helical jet model when attempting to explain large misalignments (Cassaro et al. 2002, and references therein). Under such circumstances the concept of restarted activity, coupled with a swift repositioning of the central engine of the radio source due to a merger event, is a very competitive alternative because of its simplicity and plausibility. As this scenario may also work very well for $\mathrm{X}$-shaped sources, they could be regarded as a parent population of CDTs; the apparent existence of the latter class is simply the result of orientation effects on X-shaped sources. More numerous and complete samples of both classes are needed to confirm this on a sound statistical basis but, at least, the scarcity of both X-shaped and CDT objects seems to qualitatively agree.

No misalignment was detected in the fourth source under investigation, TXS $0818+214$, and only a rather modest $\left(30^{\circ}\right)$ misalignment was observed in TXS $1312+563$. The inner parts of these two sources could be interpreted as double-lobed structures, but further multifrequency observations are necessary to provide support for such an interpretation. If confirmed, TXS $0818+214$ and TXS $1312+563$ would be the most compact double-double radio sources known to date. An attempt to explain their nature by the theory developed by KSR would make a modification of this theory necessary, given that the linear sizes of these two sources are below the required limit.
Acknowledgements. MERLIN is operated by the University of Manchester as a National Facility on behalf of the Particle Physics \& Astronomy Research Council (PPARC).

This research has made use of the NASA/IPAC Extragalactic Database (NED) which is operated by the Jet Propulsion Laboratory, California Institute of Technology, under contract with the National Aeronautics and Space Administration.

Use has been made of the fourth release of the Sloan Digital Sky Survey (SDSS) Archive. Funding for the creation and distribution of the SDSS Archive has been provided by the Alfred P. Sloan Foundation, the Participating Institutions, the National Aeronautics and Space Administration, the National Science Foundation, the US Department of Energy, the Japanese Monbukagakusho, and the Max Planck Society. The SDSS Web site is http://www.sdss.org/. The SDSS is managed by the Astrophysical Research Consortium (ARC) for the Participating Institutions. The Participating Institutions are The University of Chicago, Fermilab, the Institute for Advanced Study, the Japan Participation Group, The Johns Hopkins University, Los Alamos National Laboratory, the MaxPlanck-Institute for Astronomy (MPIA), the Max-Planck-Institute for Astrophysics (MPA), New Mexico State University, University of Pittsburgh, Princeton University, the United States Naval Observatory, and the University of Washington.

A.M. acknowledges the receipt of a travel grant funded by RadioNet as a part of the Trans-National Access (TNA) programmes.

We thank Tom Muxlow for help with data reduction.

\section{References}

Appl, S., Sol, H., \& Vicente, L. 1996, A\&A, 310, 419

Alexander, P., \& Leahy, J. P. 1987, MNRAS, 225, 1

Attridge, J. M., Roberts, D. H., \& Wardle, J. F. C. 1999, ApJ, 518, L87

Barth, A. J. 2003, Carnegie Observatories Astrophysics Series,

1: Coevolution of Black Holes and Galaxies, ed. L. C. Ho [arXiv: astro-ph/0310436]

Bardeen, J. M., \& Petterson, J. A. 1975, ApJ, 195, L65

Becker, R. H., White, R. L., \& Edwards, A. L. 1991, ApJS, 75, 1 van Breugel, W., Heckman, T., \& Miley, G. 1984, ApJ, 276, 79

Capetti, A, Zamfir, S., \& Rossi, P. 2002, A\&A, 394, 39

Cassaro, P., Stanghellini, C., Dallacasa, D., Bondi, M., \& Zappalà, R. A. 2002, A\&A, 381, 378

Cohen, A. S., Röttgering, H. J. A., Jarvis, M. J., Kassim, N. E., \& Lazio, T. J. W. 2004, ApJS, 150, 417

Cohen, A. S., Clarke, T. E., Feretti, L., \& Kassim, N. E. 2005, ApJ, 620, L5

De Breuck, C., van Breugel, W., Röttgering, H. J. A., \& Miley, G. 2000, A\&AS, 143, 303

Dennett-Thorpe, J., Scheuer, P. A. G., Laing, R. A., et al. 2002, MNRAS, 330, 609

Fanaroff, B. L., \& Riley, J. M. 1974, MNRAS, 167, 31

Hargrave, P. J., \& Ryle, M. 1974, MNRAS, 166, 305

Hatziminaoglou, E., Siemiginowska, A., \& Elvis, M. 2001, ApJ, 547, 90

Hewett, P. C., Foltz, C. B., \& Chaffee, F. H. 1995, AJ, 109, 1498

Kaiser, C. R., Schoenmakers, A. P., \& Röttgering, H. J. A. 2000, MNRAS, 315, 381 (KSR)

Komissarov, S. S., \& Gubanov, A. G. 1994, A\&A, 285, 27

Kunert-Bajraszewska, M., Marecki, A., Thomasson, P., \& Spencer, R. E. 2005, A\&A, 440, 93

Kunert-Bajraszewska, M., Marecki, A., \& Thomasson, P. 2006, A\&A, accepted 
Lara, L., Márquez, I., Cotton, W. D., et al. 1999 A\&A, 348, 699

Lara, L., Cotton, W. D., Feretti, L., et al. 2001, A\&A, 370, 409

Liu, R., Pooley, G. G., \& Riley, J. M. 1992, MNRAS, 257, 545

Liu, F. K., Wu, X., \& Cao, S. L. 2003, MNRAS, 340, 411

Liu, F. K. 2004, MNRAS, 347, 1357

Mack, K.-H., Vigotti, M., Gregorini, L., et al. 2005, A\&A, 435, 863

Marecki, A., Barthel, P. D., Polatidis, A., \& Owsianik, I. 2003, PASA 20,16

Marecki, A., Kunert-Bajraszewska, M., \& Spencer, R. E. 2006, A\&A, in press [arXiv: astro-ph/0512034]

Merritt, D., \& Ekers, R. D. 2002, Science, 297, 1310

Murphy, D. W., Browne, I. W. A., \& Perley, R. A. 1993, MNRAS, 264, 298

Pasquini, L., Bonifacio, P., Randich, S., Galli, D., \& Gratton, R. G. 2004, A\&A, 426, 651

Rottmann, H. 2001, Ph.D. Thesis, Bonn University

Röttgering, H. J. A., Lacy, M., Miley, G. K., Chambers, K. C., \& Saunders, R. 1994, A\&AS, 108, 79

Saripalli, L., Subrahmanyan, R., \& Udaya Shankar, N. 2002, ApJ, 565, 256
Saripalli, L., Subrahmanyan, R., \& Udaya Shankar, N. 2003, ApJ, 590, 181

Saripalli, L., Hunstead, R. W., Subrahmanyan, R., \& Boyce, E. 2005, AJ, 130, 896

Scheuer, P. A. G. 1995, MNRAS, 277, 331

Schoenmakers, A. P., Bruyn, A. G., Röttgering, H. J. A., \& van der Laan, H. 1999, A\&A, 341, 44

Schoenmakers, A. P., Bruyn, A. G., Röttgering, H. J. A., et al. 2000a, MNRAS, 315, 371

Schoenmakers, A. P., Bruyn, A. G., Röttgering, H. J. A., van der Laan, H., \& Kaiser, C. R. 2000b, MNRAS, 315, 395

Schoenmakers, A. P., Mack, K.-H., Bruyn, A. G., et al. 2000c, A\&AS, 146, 293

Slee, O. B., Roy, A. L., Murgia, M., Andernach, H., \& Ehle, M. 2001, AJ, 122, 1172

Thompson, D., Djorgovski, S., Vigotti, M., \& Grueff G., 1994, AJ, 108,828

Wang, T., Zhou, H., \& Dong, X. 2003, AJ, 126, 113

White, R. L., Becker, R. H., Helfand, D. J., \& Gregg, M. D. 1997, ApJ, 475,479 